\title{
MeuSIGA: Uma Aplicação de Desenvolvimento por Usuário Final em um Sistema Integrado de Gestão Acadêmica
}

\author{
Mariana Aparecida dos Santos \\ DECOM/FACET - UFVJM \\ Diamantina, Brasil \\ mariana.santos@ufvjm.edu.br
}

\author{
Maria Lúcia Bento Villela \\ DECOM/FACET - UFVJM \\ Diamantina, Brasil \\ maria.villela@ufvjm.edu.br
}

\begin{abstract}
RESUMO
A abordagem end-user development (EUD), ou desenvolvimento por usuário-final, vem sendo cada vez mais utilizada, uma vez que tem intensificado o número de pessoas, com diferentes níveis de conhecimento em computação, que desenvolvem software para apoiá-las na realização de tarefas específicas. Neste contexto, o presente trabalho apresenta uma ferramenta de programação visual de apoio ao EUD, baseada nos resultados de uma Revisão Sistemática da Literatura (SLR). Tal ferramenta se propõe a oferecer aos alunos da Universidade Federal do Vales do Jequitinhonha Mucuri (UFVJM) a possibilidade de customizar a interface do módulo ensino do se Sistema Integrado de Gestão Acadêmica (SIGA), de forma a ajustá-la aos seus interesses.
\end{abstract}

\section{Palavras-chaves}

Desenvolvimento por Usuário-Final; Programação por Usuário-Final; Sistema Integrado de Gestão Acadêmica

\section{ACM Classification Keywords}

H.5.m. Information Interfaces and Presentation (e.g. HCI): Miscellaneous

\section{INTRODUÇÃO}

O uso de aplicações de software em diferentes contextos, seja para a realização de tarefas de trabalho ou para fins de entretenimento, tornou-se uma tendência nos últimos anos [1]. Nesse sentido, tem-se intensificado o número de pessoas, com pouco ou nenhum conhecimento em programação, que personalizam os softwares para apoiá-las na realização de tarefas específicas, tornando-se assim designers de suas próprias aplicações [2].

Tal fato caracteriza a abordagem de desenvolvimento denominada End-User Development (EUD), ou Desenvolvimento por Usuário Final. Esta abordagem pode ser definida como um conjunto de métodos, técnicas e ferramentas que possibilitam aos usuários não profissionais atuarem como desenvolvedores de software, criando, modificando ou ampliando softwares para atingirem um objetivo [3].

\footnotetext{
Permission to make digital or hard copies of all or part of this work for personal or classroom use is granted without fee provided that copies are not made or distributed for profit or commercial advantage and that copies bear this notice and the full citation on the first page. Copyrights for components of this work owned by others than the author(s) must be honored. Abstracting with credit is permitted. To copy otherwise, or republish, to post on servers or to redistribute to lists, requires prior specific permission and/or a fee. Copyright 2018 SBC.

IHC 2018, Anais Estendidos do XVII Simpósio Brasileiro sobre Fatores Humanos em Sistemas Computacionais.

Outubro 22-26, 2018, Belém, Brasil

PÔSTERES VIRTUAIS E DEMOS
}

Neste contexto, é proposta uma ferramenta que utiliza a abordagem de EUD, denominada MeuSIGA. Tal ferramenta possibilita que o usuário personalize o Sistema Integrado de Gestão Acadêmica (SIGA), da Universidade Federal dos Vales do Jequitinhonha e Mucuri (UFVJM). A motivação para o desenvolvimento da referida ferramenta é que o SIGA possui um grande número de funcionalidades em sua interface, que nem sempre são utilizadas pelos seus usuários, podendo assim tornar ineficiente a sua interação com o sistema.

\section{A PROPOSTA DO MEUSIGA}

O MeuSIGA consiste em uma solução de EUD voltada para domínio específico de gestão acadêmica, utilizando o estilo de interação de programação visual. Tal solução destina-se aos discentes da UFVJM e possibilita a personalização da interface do módulo Ensino do seu Sistema Integrado de Gestão Acadêmica (SIGA), de forma a ajustá-la aos interesses dos seus usuários.

Em razão de limitações quanto a estrutura tecnológica da instituição, que atualmente não possibilita que sites sejam hospedados em seu domínio, a ferramenta foi desenvolvida para o ambiente desktop. Dessa forma, o MeuSIGA consiste numa extensão para o navegador Google Chrome, e foi desenvolvido utilizando as linguagens Javascript, HTML e CSS. Tal aplicação permite que os alunos atuem como designers do sistema acadêmico SIGA, adequando-o às suas necessidades.

O delineamento das funcionalidades que compõem o MeuSIGA se deu a partir de um mapeamento que inicialmente identificou as funcionalidades principais do SIGA. Em um segundo momento, para cada uma dessas funcionalidades, foram identificadas as sub-funcionalidades e seus respectivos links (responsável pelo redirecionamento do usuário da MeuSIGA para o sistema SIGA). A partir dessas informações, foi elaborada uma tabela que contém os caminhos possíveis que o usuário consegue percorrer no sistema. Esse material foi utilizado como guia para a construção dos elementos presentes na interface do MeuSIGA.

\section{Design}

Com o objetivo de facilitar a interação do usuário com o sistema, o design da interface do MeuSIGA foi desenvolvido para oferecer maior visibilidade das opções disponíveis para uso do aluno, bem como reduzir os caminhos de interação entre o estado atual e o objetivo do usuário no sistema. Dessa forma, as funcionalidades foram dispostas em abas em sua interface, onde estão presentes as sub-funcionalidades associadas a cada 
uma delas, como ilustrada na Figura 1. Assim, os usuários conseguem visualizar todas as opções existentes com mais facilidade e a um custo de interação menor, navegando pelas abas disponíveis.

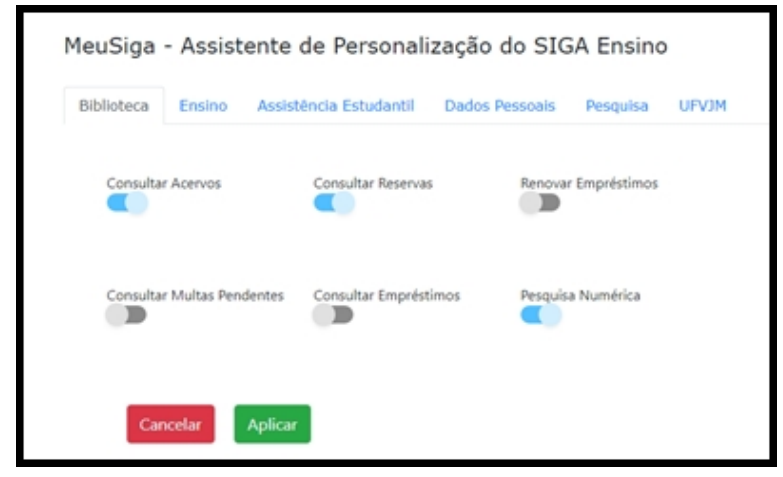

Figure 1. Interface do MeuSIGA

Como pode ser visto na Figura 1, a interface do MeuSIGA é composta por caixas de seleção com valores pré-determinados, referentes às sub-funcionalidades disponíveis no SIGA Ensino, permitindo então que o usuário exerça o papel de designer sobre o sistema, aplicando-se então técnicas de programação visual. Desse modo, para personalizar a interface do sistema, o usuário escolhe, dentre as opções disponíveis, as que são necessárias para cumprir seus objetivos. Em seguida, as opções que não foram selecionadas são ocultadas da interface, uma vez que o MeuSIGA interpreta o conjunto de valores escolhidos como sendo as únicas opções que o usuário gostaria de visualizar no sistema. Essas opções são oferecidas como ícones, como apresentado na Figura 2, que atuam como gatilhos para que o usuário acesse a funcionalidades específicas do SIGA.

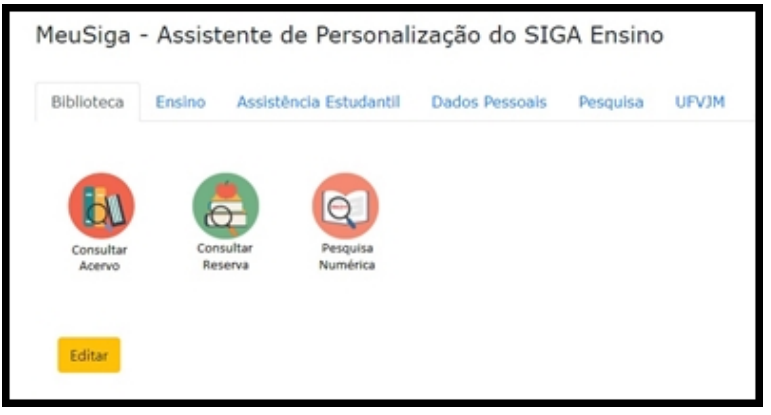

Figure 2. Ícones da interface do MeuSIGA

\section{Avaliação}

A preocupação com a qualidade de uso do MeuSIGA esteve presente durante o seu design, ao buscar uma interface simples, seguindo diretrizes de usabilidade [4]. Assim, a fim de verificar fatores de usabilidade e experiência de uso, foi realizada uma avaliação com oito usuários, refletindo os perfis dos discentes da UFVJM.

Com base na análise dos dados levantados durante os testes com usuários, foi possível concluir que o MeuSIGA atendeu ao propósito para que foi desenvolvido, ou seja permitir que os discentes personalizem o sistema acadêmico de acordo com suas necessidades, melhorando seu desempenho quanto ao uso da mesma.

Embora os participantes tenham apresentado dificuldades durante a realização de algumas tarefas, os problemas identificados possuem um baixo nível de gravidade e podem ser facilmente superados pelo usuário à medida que for utilizando a ferramenta. No sentindo de facilitar a interação, foi desenvolvido um tutorial, em atendimento à sugestão dada por um dos participantes.

\section{CONCLUSÃO E TRABALHOS FUTUROS}

A motivação para o desenvolvimento da MeuSIGA se deu ao identificar que a interface atual do SIGA Ensino oferecia aos usuários um grande número de informações, em sua maioria desnecessárias, o que tornava sua interação ineficiente.

Assim, o MeuSIGA consiste em uma ferramenta de apoio ao EUD, que se apresenta na forma de uma extensão para navegadores, permitindo que seus usuários atuem como designers do SIGA Ensino da UFVJM, personalizando-o de acordo com suas necessidades. Os resultados da avaliação com usuários do MeuSIGA mostraram que ele atendeu o seu principal objetivo de tornar mais fácil a interação do usuário com o sistema de gestão acadêmica da universidade, refletindo assim no aumento da sua eficiência de uso. Como próximo passo do trabalho, pretende-se fazer uma avaliação mais ampla da ferramenta, abordando outras qualidades de uso. Além disso, pretende-se estudar a viabilidade de integrar tal ferramenta ao sistema acadêmico utilizado pela universidade, permitindo assim que as escolhas dos usuários sejam feitas pela própria ferramenta, com base no histórico de uso dos alunos.

\section{REFERÊNCIAS}

1. G Ghiani, F Paternò, L D Spano, and G Pintori. 2016. An environment for End-User Development of Web mashups. International Journal of Human-Computer Studies 87 (mar 2016), 38-64. DOI : http://dx.doi.org/10.1016/j.ijhcs.2015.10.008

2. A J Ko, B Myers, M B Rosson, G Rothermel, M Shaw, S Wiedenbeck, R Abraham, L Beckwith, A Blackwell, M Burnett, M Erwig, C Scaffidi, J Lawrance, and H Lieberman. 2011. The state of the art in end-user software engineering. Comput. Surveys 43, 3 (2011), 1-44. DOI : http://dx . doi .org/10.1145/1922649.1922658

3. H Lieberman, F Paternò, M Klann, and V Wulf. 2006. End-User Development: An Emerging Paradigm. In End User Development. Springer Netherlands, Dordrecht, 1-8. DOI : http://dx.doi .org/10.1007/1-4020-5386-X_1

4. Jakob Nielsen. 1994. Usability inspection methods. In Conference companion on Human factors in computing systems. ACM, 413-414. 